\title{
Systemic polyclonal immunoblastic proliferation: a diagnostic review and differential diagnosis
}

\author{
Adeel Raza • David Cohen • Sapna Amin • \\ Arthur Zieske • April Ewton
}

Received: 17 February 2014 / Accepted: 28 May 2014 /Published online: 7 June 2014

(C) Springer-Verlag Berlin Heidelberg 2014

\begin{abstract}
Systemic polyclonal immunoblastic proliferation (SPIP) is a very rare, atypical expansion of plasma cells and lymphoblasts associated with an aggressive clinical presentation. The pathogenesis of SPIP is unknown and it is unclear whether these proliferations represent a clinicopathologic entity or rather a pattern of cellular proliferation. Some evidence of infection as an initiating event has been proposed. SPIP is often difficult to differentiate from other disorders, particularly in the context of an obscuring inflammatory response. Untreated or even treated cases can be fatal. The morphologic features are characterized by a pronounced proliferation of B immunoblasts and plasma cells that typically involve the blood, bone marrow, lymph nodes, spleen, thymus, liver, kidneys, thyroid, adrenal glands, and lungs. A subset of reported cases has responded well to treatment with corticosteroids alone or in combination with cytotoxic agents, showing complete remission and resolution of lymphadenopathy and normalization of peripheral blood findings. Here, we present a case of SPIP in a 58-year-old male with polyclonal plasmacytosis, hypergammaglobulinemia, diffuse lymphadenopathy, splenomegaly, and a diffuse maculopapular rash; SPIP with skin involvement is very unusual. Fortunately, our patient showed a striking response to steroids. This article provides a brief overview of the clinicopathologic features associated with this atypical lymphoplasmacytic proliferation and a review of the literature highlighting the differential diagnosis of lymphoplasmacytic disorders.
\end{abstract}

Keywords SPIP · Plasma cells $\cdot$ Immunoblasts

A. Raza $(\bowtie) \cdot$ D. Cohen $\cdot$ S. Amin $\cdot$ A. Zieske $\cdot$ A. Ewton Department of Pathology and Genomic Medicine, Houston Methodist Hospital, 6565 Fannin St, M227, Houston, TX 77030, USA

e-mail: adeelrazamd@hotmail.com

\section{Introduction}

Infiltrative processes with prominent plasma cell and immunoblast populations are rare and may be seen in a variety of settings. Systemic polyclonal immunoblastic proliferation (SPIP) was first defined by Peterson et al. [1] in 1988 as a benign polyclonal proliferation of plasma cells and immunoblasts. SPIP represents a constellation of clinical and histologic findings in cases for which no definitive etiology can be identified. As such, SPIP is a very rare condition; to the best of our knowledge, fewer than 15 cases have been reported in the literature. It is characterized by a pronounced proliferation of B immunoblasts and plasma cells that typically involves the blood, bone marrow (BM), and lymph nodes with rare cases involving the spleen, thymus, liver, kidneys, thyroid, adrenal glands, and lungs. The most common clinical presentation is fever with leukocytosis. The peripheral blood (PB) may also show increased numbers of B immunoblasts and plasma cells. Other presenting features include lymphadenopathy, hepatosplenomegaly, and jaundice, along with other systemic symptoms similar to malignant lymphoma, multiple myeloma, and autoimmune diseases. These findings generate a differential diagnosis including other processes that show nodal infiltration of immunoblasts and plasma cells, such as viral lymphadenopathy or infectious mononucleosis, and autoimmune diseases such as rheumatoid arthritis. In a few cases, SPIP has been reported to be associated with bacterial infections [2]. Rare cases have cytogenetic abnormalities [1].

\section{Clinical history}

A 58-year-old man was admitted to the hospital with a chief complaint of extreme fatigue, intermittent fevers, and tachypnea. Clinical examination was remarkable for diffuse lymphadenopathy, splenomegaly, and nonpruritic 
maculopapular rash most pronounced on his back. His past medical and surgical history was unremarkable with no history of HIV, malignancy, infection, or travel outside of the USA. CBC revealed a WBC count of $5.23 \mathrm{~K} / \mu \mathrm{L}(4.50-11.00), \mathrm{RBC}$ of $1.26 \mathrm{~m} / \mu \mathrm{L}(4.40-6.00)$, hemoglobin of $4.1 \mathrm{~g} / \mathrm{dL}(14.0$ 18.0 ), hematocrit of $11.4 \%$ (41-51), and platelet count of $19 \mathrm{~K} / \mu \mathrm{L}(150-400)$. Laboratory findings for infectious etiology were non-diagnostic with negative cultures and serology for HIV, CMV, HSV, hepatitis B and C, EBV, and AFB. CSF studies for fungal diseases including Aspergillus, Blastomyces, Histoplasma, and Toxoplasma came back negative. Coccidioidomycosis titers were found at an outside hospital to be elevated to 1:1,024 but repeat serologies were negative. MRI of the head was performed which showed no evidence of enhancement. Serum protein electrophoresis showed a polyclonal hypergammaglobulinemia. Cold agglutinin titers were positive, at a 1:32 dilution. A lymph node biopsy performed at an outside institution showed reactive hyperplasia with no evidence of lymphoma. A peripheral blood smear was reviewed, and flow cytometry was performed on peripheral blood. Serologic workup for autoimmune disease was negative. Bone marrow biopsy was performed to rule out underlying malignancy.

\section{Materials and methods}

Wright-Giemsa-stained peripheral blood, bone marrow aspirate smears, aspirate clot, and core biopsy along with skin biopsy were received. Sections from skin biopsy were fixed in B5 and $10 \%$ neutral buffered formalin. The bone marrow biopsy was fixed in B5 and formalin with subsequent decalcification. Specimens were routinely processed and embedded in paraffin using routine histological techniques. Sections were stained with hematoxylin and eosin. Bone marrow aspirate preparations were stained with Wright-Giemsa stain.

Immunohistochemistry and in situ hybridization for light chains

Immunohistochemical stains were performed on 5- $\mu \mathrm{m}$ paraffin sections of formalin-fixed, paraffin-embedded tissues using automated immunostainer (Ventana Medical Systems, Tuscon, AZ, USA). Immunohistochemical stains used were CD3, CD20, CD21, MUM-1, IgM, IgG, Ki-67, and CD138. In situ hybridization was performed using a probe complimentary to the Epstein-Barr virus (EBV)-encoded nonpolyadenylated RNA transcript: EBER1 (Ventana Medical System, Tucson, AZ). Immunoglobulin kappa and lambda light chains were performed using oligonucleotide probes labeled with a fluorescein conjugate in a formamide-based diluent (inform cytoplasmic immunoglobulin $\mathrm{K}$ and $\mathrm{L}$, Ventana). The intended target is cytoplasmic immunoglobulin kappa or lambda light chains mRNA. Sections of benign tonsil were used for positive controls.

Immunophenotypic analysis by flow cytometry

Eight color flow cytometry immunophenotypic analysis was performed on $\mathrm{PB}$ and $\mathrm{BM}$ aspirate. The panel of monoclonal antibodies included reagents for $\mathrm{CD} 2$ (fluorescein isothiocyanate, FITC), cytoplasmic CD3 (V450), CD4 (allophycocyanin, APC), CD5 (FITC), CD7 (phycoerythrin, PE), CD8 (peridin-chlorophyll-a-protein, PerCP-Cy5.5), CD10 (PE), CD11b (APC), CD13 (PE), CD14 (PE Cy7), CD16 (APC Cy7/H7), CD19 (PE), CD20 (FITC), CD33 (PE), CD34 (PerCP), CD38 (FITC), CD45 (PerCP), CD56 (V450), CD64 (PE), CD71 (PerCP), CD117 (PE), CD123 (PerCP), CD138 (PE), monoclonal kappa (APC), monoclonal lambda (FITC), polyclonal kappa (PE), and polyclonal lambda (FITC). The antibodies were purchased from BD Biosciences and the analysis was performed using FACS Diva.

Conventional cytogenetic analysis

Conventional cytogenetic karyotyping was performed on metaphase cells from BM aspirate cultures for $24 \mathrm{~h}$ without mitogens using standard techniques. Giemsa-banded metaphases were analyzed and the results were reported using the International System for Human Cytogenetic Nomenclature, 2005.

\section{Results}

Peripheral blood findings

The peripheral blood showed normochromic normocytic anemia with rouleaux formation and aggregation and increased plasma cells (Fig. 1). There were scattered reactive lymphocytes and plasmacytoid lymphocytes. Platelets were decreased in number and had normal morphology. Flow cytometry performed on the PB showed $10 \%$ polytypic plasma cells with no aberrant immunophenotype.

Bone marrow findings

The BM was $90 \%$ cellular (Fig. 2a), and large clusters of small and mature plasma cells were seen (Fig. 2b). Immunohistochemical stains confirmed the presence of large aggregates of CD138-positive plasma that comprised approximately $25 \%$ of marrow cellularity. These plasma cells were polytypic by in situ hybridization for kappa and lambda light chains (Fig. 2c, d). The polytypic nature of the plasma cells correlated well with the presence of polyclonal 


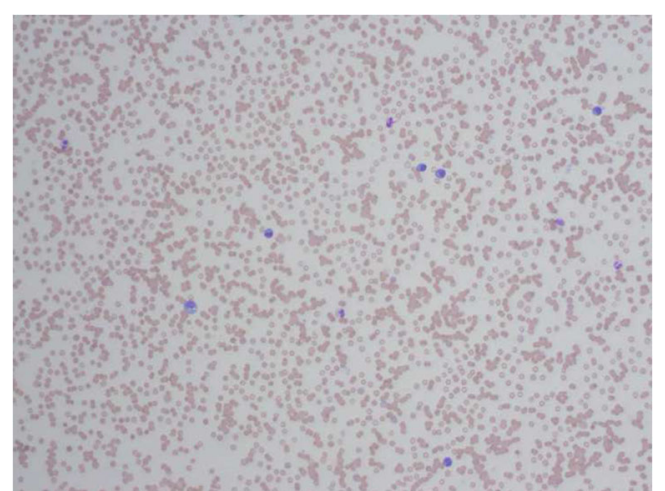

Fig. 1 Peripheral blood smear. Increased in plasma cells with rouleaux formation and aggregation

hypergammaglobulinemia and the absence of a clonal paraprotein by serum protein electrophoresis. CD20 showed few scattered B cells in a normal distribution. CD21, IgG, and EBER by in situ hybridization were negative. IgM was expressed by small subset of plasma cells. MUM-1 positive plasma cells showed a very low proliferation index by Ki-67 staining (Fig. 3a-d). The remaining marrow showed erythyroid hyperplasia with mild dyspoiesis. Special stains for fungal infection were negative. Corresponding flow cytometry performed on the BM aspirate detected $34 \%$ polytypic plasma cells and $2 \%$ lambda light chain-restricted B cells negative for CD5 and CD10. Cytogenetic studies showed a normal male karyotype.

\section{Skin findings}

Skin punch biopsy of the left abdomen showed a prominent perivascular and periadnexal infiltrate involving the superficial dermis (Fig. 4a). The infiltrate was comprised of predominantly atypical plasmacytoid cells that were enlarged with conspicuous nucleoli, intracytoplasmic immunoglobulin deposition, and frequent mitoses (Fig. 4b). Rare Dutcher bodies and immunoblastic cells were also present. These cells were confirmed to be polyclonal and showed no light chain restriction for either kappa or lambda by immunohistochemistry or in situ hybridization. CD20 highlights scattered B cells (Fig. 4c). CD21, IgG, and EBER by in situ hybridization were negative. A small proportion of plasma cells were positive for IgM. Ki-67 proliferation rate was low in the MUM-1 positive plasma cells (Fig. 4c).

\section{Clinical management and follow-up}

On day 5 post-admission, the patient developed acute kidney failure, elevated liver enzymes, continuing bicytopenia, and complete vision loss in his right eye, thought to be due to ischemic optic neuropathy. Unfortunately, blood products including platelets and packed red blood cells could not be given due to the patient's religious affiliation. The patient was initially started on erythropoietin, intravenous immunoglobulin, and low-dose steroids with little improvement. Rituximab was withheld due to his tenuous status. Eight days after his admission, the patient responded to an initiation of high-dose steroids, with a slow increase over a 1-week period in hemoglobin to $9.5 \mathrm{~g} / \mathrm{dL}$ and platelets to $91 \mathrm{~K} / \mu \mathrm{L}$. Due to his strong response to steroids with resolution of weakness and fatigue, and improvement of maculopapular rash and lymphadenopathy, a repeat lymph node biopsy was not performed. The patient was discharged with instructions for close follow-up care by his hematologist.
Fig. 2 Bone marrow biopsy. a Hypercellular marrow. b Higher magnification shows clusters of mature plasma cells, few lymphocytes, and immunoblasts. c, d Kappa and lambda by in situ hybridization show similar numbers
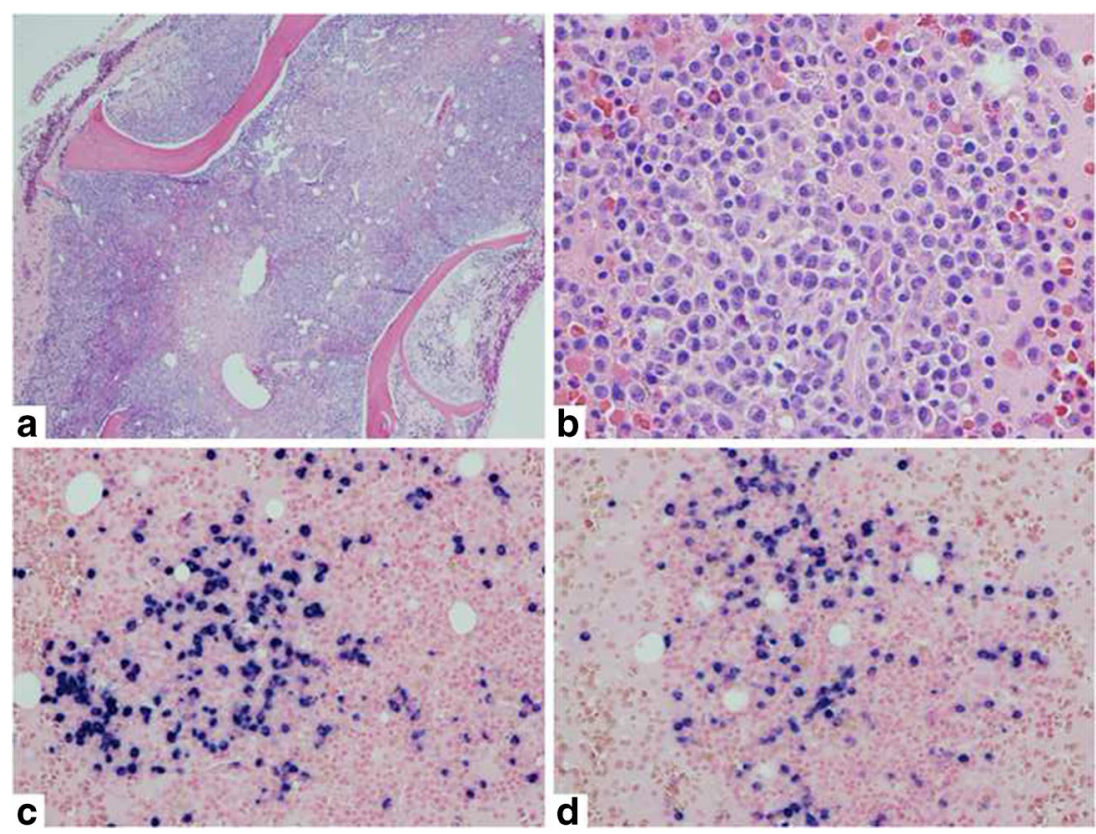
Fig. 3 Bone marrow immunostains. a CD20 highlights few $B$ cells. $\mathbf{b}$ CD21 is negative. $\mathbf{c}$ $\mathrm{IgG}$ is negative in plasma cells. $\mathbf{d}$ Small subset of plasma cells are positive for IgM
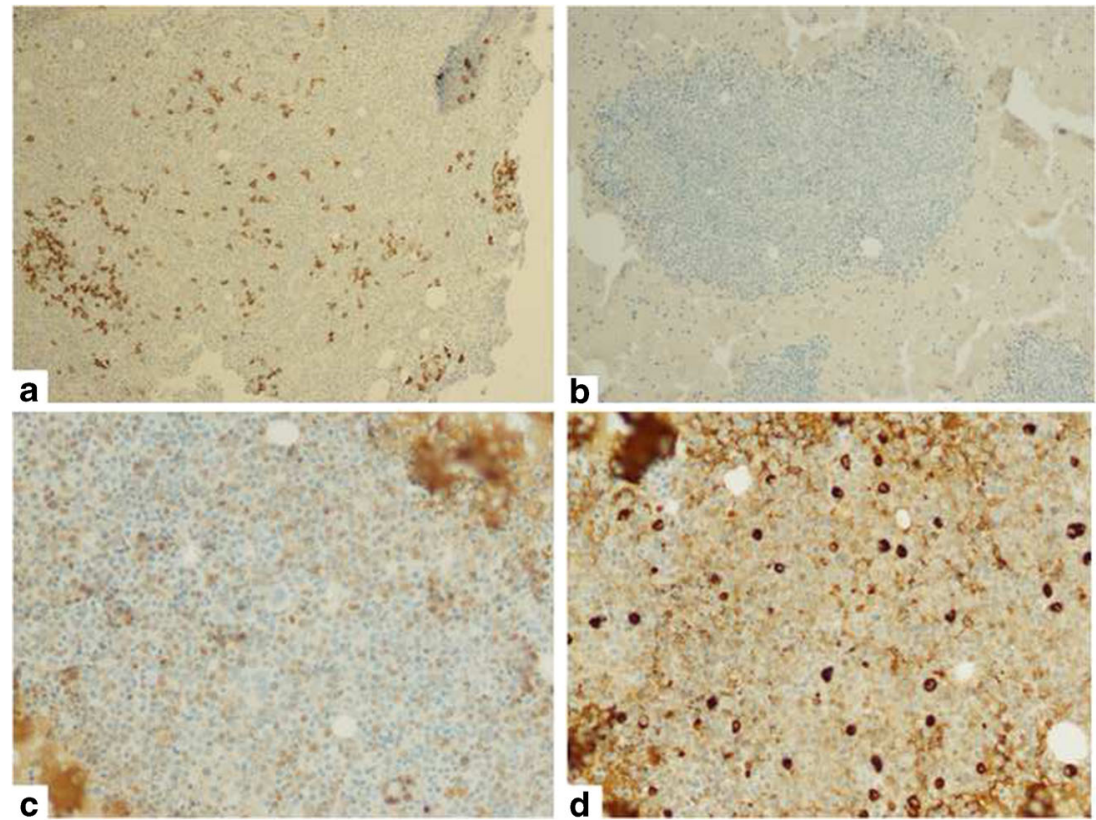

\section{Discussion}

In this case, the increase of plasma cells in the PB and BM is a worrisome finding suggestive of a plasma cell malignancy. However, immunophenotyping by flow cytometry and immunohistochemistry identified the plasma cells as a polyclonal population consistent with a benign reactive process rather than a malignant monoclonal process. The polyclonality was further supported by immunoelectrophoresis of serum protein that showed a prominent polyclonal gammopathy without an M-spike. BM plasmacytosis has been described previously in various inflammatory conditions, chronic infections, autoimmune diseases, and hypersensitivity reactions [3]. The normal upper limit for BM plasma cells is approximately $2 \%$. BM plasmacytosis ranging from 10 to $20 \%$ have been reported in association with septic arthritis, systemic lupus erythematosus, and cirrhosis [4] but percentages exceeding $20 \%$ of cells is extremely rare; however, Holbert described a case in 1988 in which marrow plasma cells exceeded $40 \%$ after the patient received rabies vaccine [5].

SPIP shares features with other lymphoproliferative disorders and the lymph nodes from these patients can show reactive hyperplasia of germinal centers along with increased plasma cells mimicking Castleman disease. In the 1980s, Mori
Fig. 4 Skin biopsy. a

Perivascular and periadnexal infiltrate involving the superficial dermis. b Infiltrate predominantly composed of plasma cells. c CD20 is positive in few scattered B cells. d Ki-67 has increased activity overall but is low in MUM1-positive plasma cells
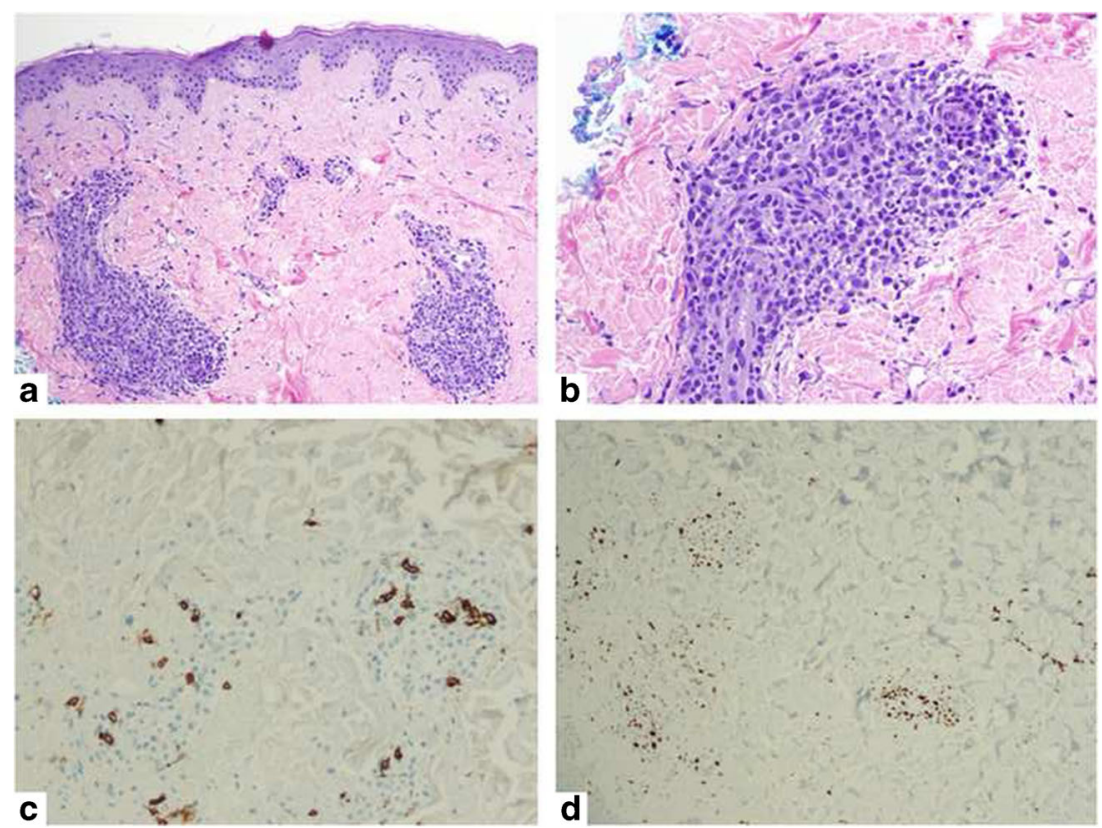
and coworkers [6] identified markedly increased plasma cells in reactive lymph nodes of patients having polyclonal hyperimmunoglobulinemia, with histological findings similar to a plasma cell variant of multicentric Castleman disease (MCD). Later, Fizzera and colleagues [7, 8] identified idiopathic polyclonal lymphadenopathy identical to MCD in 15 patients. Clinically, the patients presented with diffuse lymphadenopathy, polyclonal hypergammaglobulinemia and BM plasmacytosis; however, their study showed that MCD exhibits an aggressive clinical course associated with infectious complications and increased risk of malignancies such as Kaposi sarcoma and B cell lymphomas which was not seen in patients with idiopathic polyclonal lymphadenopathy. Also morphologically the lymphoid follicles have hyaline vascular germinal centers in MCD compared to normal or hyperplastic germinal centers of idiopathic polyclonal lymphadenopathy.

Popadi et al. [9] reported two cases of angioimmunoblastic T cell lymphoma (AITL) that initially presented with unexplained, massive systemic B immunoblastic and plasmacytic proliferation mimicking SPIP. PB in these two patients revealed numerous immunoblasts and plasma cells. BM aspirates and biopsies were hypercellular with diffuse infiltration by immunoblasts and plasma cells. The cervical lymph node biopsy in the first patient showed partial effacement of nodal architecture by interfollicular proliferation of B immunoblasts and plasma cells, with the remaining follicles having hyperplastic germinal centers. No increase in vascularity was seen. Flow cytometry showed polytypic plasma cells with no aberrant $\mathrm{T}$ cells. However, clonal TCR gene rearrangement was detected in the lymph node. The inguinal lymph node biopsy in the second patient showed architectural effacement by complex lymphoid nodules of variable size composed of lymphoid cells, immunoblasts, and plasma cells. Rare atypical cells had clear cytoplasm. The flow cytometry revealed a predominance of $\mathrm{T}$ cells, few polyclonal B cells, and plasma cells and a small population of CD4-positive cells with a weaker expression of CD3. Clonal TCR gene rearrangement was detected in the lymph node. The diagnosis of AITL in these two cases was difficult due to the presence of numerous polyclonal B immunoblasts and plasma cells within the blood and $\mathrm{BM}$. Clonal $\mathrm{T}$ cell gene rearrangement and immunohistochemical staining showed increased expression of PD1/ CD279, which was useful in diagnosing both cases.

The pathogenesis of plasma cell and immunoblastic infiltrations is unclear. Peterson and colleagues [1] suggested evidence of an acute immune disorder in SPIP. It is unclear whether an altered immune status may have predisposed patients to infection in those cases associated with bacterial infections, whether the infections may have initiated an atypical immune response, or whether the association was incidental.

Cytogenetic analyses of BM on two patients in Peterson's study [1] detected a chromosomal translocation involving band $14 \mathrm{q} 32$. The authors postulated that this phenomenon may represent the polyclonal phase of an evolving B cell lymphoma. The proliferation of plasma cells and immunoblasts in these patients also raised the possibility of an early B cell lymphoma with an associated polyclonal B cell proliferation or an intermediate step in progression from polyclonal to a monoclonal phase. Transformation to lymphoma in this case may have been prevented with the administration of steroids. Interestingly, flow cytometry performed on the bone marrow from this patient showed $2 \%$ lambda light chainrestricted B cells.

Disorders involving infiltration by immunoblasts and abundant plasma cells are rare. SPIP as described here and elsewhere in the literature may be a clinicopathologic entity; however, until a distinct etiology or pathologic mechanism of disease can be delineated, a broad differential diagnosis should be considered in the evaluation of such cases. If no specific etiology can be determined, SPIP is the diagnosis of exclusion. Treatment of SPIP patients is mainly symptomatic and supportive. In a few reported cases, patients showed complete resolution of peripheral plasmacytosis with the administration of steroids [10]. Deaths have been reported in some cases but were largely associated with comorbid conditions such as sepsis [11].

Although the initial finding of marked peripheral blood or bone marrow plasmacytosis suggests a plasma cell neoplasm, pathologists must remain aware of benign processes with associated plasmacytosis. Ancillary studies that will aid in confirming a polyclonal plasma cell infiltrate include flow cytometric immunophenotyping, immunohistochemical stains or in situ hybridization for kappa and lambda light chains, immunoglobulin gene rearrangement studies by PCR, and serum electrophoresis.

Conflict of interest The authors declare that they have no conflict of interest.

\section{References}

1. Peterson LC, Kueck B, Arthur DC et al (1988) Systemic polyclonal immunoblastic proliferations. Cancer 61(7):1350-1358

2. Poje EJ, Soori GS, Weisenburger DD (1992) Systemic polyclonal Bimmunoblastic proliferation with marked peripheral blood and bone marrow plasmacytosis. Am J Clin Pathol 98(2):222-226

3. Kojima M, Murayama K, Igarashi T et al (2007) Bone marrow plasmacytosis in idiopathic plasmacytic lymphadenopathy with polyclonal hyperimmunoglobulinemia - a report of four cases. Pathol Res Pract 203(11):789-794

4. Hyun BK, Kwa D, Herminia G et al (1976) Reactive plasmacytic lesions of the bone marrow. Am J Clin Pathol 65(6):921-927

5. Holbert JM (1988) Neoplasms of terminally differentiated B lymphocytes. Clin Lab Med 8(1):197-209

6. Mori S, Mohri N, Uchida T et al (1980) Idiopathic plasmacytic lymphadenopathy with polyclonal hyperimmunoglobulinemia; a 
syndrome related to giant lymph node hyperplasia of plasma cell type. J Jpn Soc Res 20:55-65

7. Fizzera G, Massarelli G, Banks P et al (1983) A systemic lymphoproliferative disorder with morphologic features of Castleman's disease. Pathologic findings in 15 patients. Am J Surg Pathol 7(3):346364

8. Frizzera G, Peterson B, Bayrd E et al (1985) A systemic lymphoproliferative disorder with morphologic features of Castleman's disease: clinical findings and clinicopathologic correlations in 15 patients. $\mathbf{J}$ Clin Oncol 3(9):1202-1216
9. Popadi B, Polski J, Clarkson D (2012) Atypical angioimmunoblastic T-cell lymphomas masquerading as systemic polyclonal Bimmunoblastic proliferation. Virchows Arch 461(3):323-331

10. Shtalrid M, Shvidel L, Vorst E (2003) Polyclonal reactive peripheral blood plasmacytosis mimicking plasma cell leukemia in a patient with staphylococcal sepsis. Leuk Lymphoma 44(2):379380

11. Li L, Hsu P, Patel K et al (2006) Polyclonal plasma cell proliferation with marked hypergammaglobulinemia and multiple autoantibodies. Ann Clin Lab Sci 36(4):479-484 